The INL is a

U.S. Department of Energy

National Laboratory

operated by

Battelle Energy Alliance

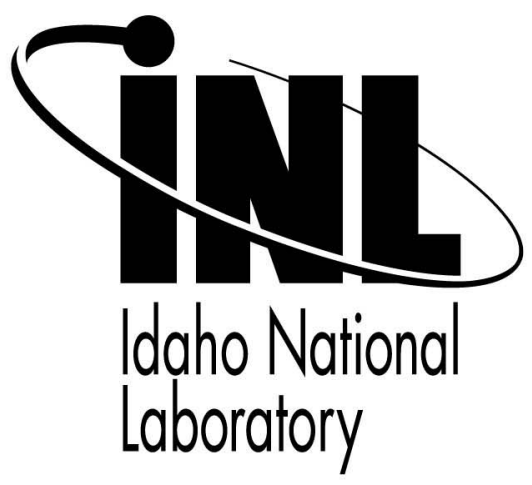

\section{Conditionally-Sampled Turbulent and Nonturbulent Measurements of Entropy Generation Rate in the Transition Region of Boundary Layers}

\section{Turbo Expo 2006 (International Gas Turbine Conference)}

Edmond J. Walsh

Kevin P. Nolan

Donald M. McEligot

Ralph J. Volino

Adrian Bejan

May 2006
This is a preprint of a paper intended for publication in a journal or proceedings. Since changes may be made before publication, this preprint should not be cited or reproduced without permission of the author. This document was prepared as an account of work sponsored by an agency of the United States Government. Neither the United States Government nor any agency thereof, or any of their employees, makes any warranty, expressed or implied, or assumes any legal liability or responsibility for any third party's use, or the results of such use, of any information, apparatus, product or process disclosed in this report, or represents that its use by such third party would not infringe privately owned rights. The views expressed in this paper are not necessarily those of the United States Government or the sponsoring agency. 


\section{Conditionally-Sampled Turbulent and Nonturbulent Measurements of Entropy Generation Rate in the Transition Region of Boundary Layers}

\section{Edmond J. Walsh}

\section{Kevin P. Nolan}

Stokes Research Institute,

Department of Mechanical and Aeronautical Engineering, University of Limerick,

Limerick, Ireland

Donald M. McEligot

Idaho National Laboratory (INL),

Idaho Falls, ID 83415-3885

\section{Ralph J. Volino}

United States Naval Academy, Department of Mechanical Engineering, Annapolis, MD 21402

\section{Adrian Bejan}

Department of Mechanical Engineering and Materials

Science,

Duke University,

Durham, NC 27708-0300

Conditionally-sampled boundary layer data for an accelerating transitional boundary layer have been analyzed to calculate the entropy generation rate in the transition region. By weighing the nondimensional dissipation coefficient for the laminarconditioned-data and turbulent-conditioned-data with the intermittency factor $\gamma$ the average entropy generation rate in the transition region can be determined and hence be compared to the time averaged data and correlations for steady laminar and turbulent flows. It is demonstrated that this method provides, for the first time, an accurate and detailed picture of the entropy generation rate during transition. The data used in this paper have been taken from detailed boundary layer measurements available in the literature. This paper provides, using an intermittency weighted approach, a methodology for predicting entropy generation in a transitional boundary layer.

Keywords: boundary layer, transition, turbulence, entropy generation

\section{Introduction}

Efficiency is one of the key concerns in the development of modern turbomachines, as modest improvements result in significant gains in cost savings and pollution reduction. The continued increases in efficiency over the years have come in part from an elevation of turbine inlet temperatures and increased pressure ratios. However, such strategies are becoming more and more difficult to implement, as the gas temperature in modern gas turbines is well in excess of the melting temperature of the blade material. Thus, other ways to increase efficiency are continually sought. To elucidate these sources, the designer must understand the characteristics and sources of various loss mechanisms. Entropy generation minimization (EGM) is a method of thermodynamic optimization of real systems that owe their thermodynamic imperfection to heat transfer, fluid flow, and mass transfer irreversibilities [1]. Denton [2] noted that entropy creation is a direct measure of lost work and is therefore the key to understanding loss mechanisms in turbomachinery flows. With the help of such knowledge it may be possible to achieve improvements of component efficiency, thereby offering advantages to the designer. Such an analysis is not restricted to turbomachinery and is applicable to all fluid flow systems.

EGM is especially important when considering the boundary layer. It is stated throughout the literature that boundary layers are a key loss generating mechanism in turbomachinery. Bejan [1] noted that for a flat plate boundary layer the near wall region, $Y^{+}<30$, is where the generation of entropy is concentrated. Much work has focused on understanding and accurately predicting both the laminar and turbulent boundary layer loss mechanisms, with reasonable success. For the laminar boundary layer Truckenbrodt [3] integrated the Pohlhausen [4] family of velocity profiles and showed that the nondimensional entropy generation rate per unit surface is inversely proportional to $\operatorname{Re}_{\theta}$. For turbulent boundary layers, Schlichting [5] demonstrated that the nondimensional entropy generation rate is only weakly dependent on the Reynolds number of the flow. Both of these results have withstood the test of time with a number of authors showing good agreement between measurements and predictions.

An important boundary layer phenomenon is the transition process from a laminar to a turbulent boundary layer, this topic being the focus of numerous experimental and theoretical investigations. The transition region may be small relative to the wetted surface at low Reynolds numbers, where the flow is predominantly laminar, and high Reynolds numbers where the flow is predominantly turbulent. However, at Reynolds and Mach numbers found in turbomachinery, through hot film measurements in a linear cascade, 
Walsh and Davies [6] demonstrated that the transition process extends over a relatively large proportion of the suction surface. The understanding and prediction of this region has plagued researchers for over a century, with an abundance of investigators addressing the prediction of transition onset and length over a diverse range of flow conditions [7-12].

The intermittent nature of transitional boundary layers has also been studied extensively, notably by Emmons [13] and Dhawan and Narasimha [14]. More recently, work on conditional sampling and intermittency has focused on the effects of free stream turbulence intensity [15-17] and passing wakes [18] on transition and the resulting structures of the transitional boundary layers. However, very limited experimental or theoretical work has been devoted to the entropy generation rate within the transition region. and no work has been presented where the transitional entropy generation rate has been calculated based on the intermittency factor $\gamma$. Stieger [19] showed ensemble-averaged and timeaveraged dissipation coefficient data for a diffusing boundary layer flow over a flat plate subject to a passing wake. It was observed that the time averaged data lie between the laminar and turbulent correlations and a somewhat erratic evolution of dissipation coefficient $C_{D}$ during transition from laminar to turbulent flow was seen.

Detailed hot wire measurements for the transition region of an accelerating boundary layer have been made available by Volino et al. [17]. This work contains nonconditionally sampled and conditionally sampled data, which consists of laminar and turbulent conditioned data in an accelerating transitional boundary layer. This means the flow within the turbulent spots and the calmed regions of the transitional boundary layer are identified first and then the data for each are separated and processed. Hence, the data are sampled based on the state of the boundary layer; laminar or turbulent. The favorable pressure gradient resulted in an extended transition region which gave improved measurement resolution in the transition region. Detailed entropy generation calculations from these data have been carried out and the conditionally-sampled data are compared with the nonconditionally-sampled data in the literature, as seen by Stieger [19]. This is the first time that dissipation has been characterized in a transitional boundary layer using the conditionally averaged approach. It should be noted that in such work care must be taken when using intermittency weighted averages of conditionally sampled data. This is because deviations of quantities identified as "laminar-like" and "turbulent-like" from actual laminar and turbulent characteristics can affect the success of the method [20]. The resultant distribution of entropy generation rate in the transition region is the first to shed light on the entropy production rate within a boundary layer as the flow transitions from a laminar to turbulent state.

\section{Entropy Generation}

The dissipation function [5] can be used to determine the volumetric entropy generation rate for steady, two-dimensional, incompressible adiabatic flows,

$$
\dot{S}_{\mathrm{gen}}^{\prime \prime \prime}=\underbrace{\frac{\mu}{T}\left[\frac{\partial \bar{u}}{\partial y}\right]^{2}}_{\begin{array}{c}
\text { viscous } \\
\text { dissipation }
\end{array}}-\underbrace{\frac{\rho \overline{u^{\prime} v^{\prime}}}{T}\left[\frac{\partial \bar{u}}{\partial y}\right]}_{\begin{array}{c}
\text { Reynolds stress } \\
\text { dissipation }
\end{array}}
$$

For laminar flow Reynolds stresses are assumed to be negligible and the second term of Eq. (1) may be ignored. It is convenient to write $\dot{S}_{\text {gen }}^{\prime \prime \prime}$ in the nondimensional form of the dissipation coefficient,

$$
C_{D}=\frac{T}{\rho u_{e}^{3}} \int_{0}^{\delta} \dot{S}_{\mathrm{gen}}^{\prime \prime \prime} d y
$$

Denton [2] noted that for turbulent boundary layers the dissipation coefficient is much less dependent on the state of the boundary layer than the more widely used skin friction coefficient, $C_{f}$ with about $90 \%$ of entropy generation occurring within the inner part of the boundary layer. Denton [2] also details a method by Truckenbrodt [3] based on an inverse relationship between dissipation coefficient and momentum thickness Reynolds number, given in Eq. (3),

$$
C_{D_{\mathrm{LAM}}}=\beta \operatorname{Re}_{\theta}^{-1}
$$

where $\beta$ has a range of $0.151 \leqslant \beta \leqslant 0.22$ for a Pohlhausen pressure gradient shape factor of $-12 \leqslant \Lambda \leqslant 12$. Schlichting [5] reports a correlation for turbulent boundary layers with a shape factor $H_{12}$ between 1.2 and 2 , and $a \operatorname{Re}_{\theta}$ between $10^{3}$ and $10^{5}$,

$$
C_{D_{\mathrm{TURB}}}=0.0056 \mathrm{Re}_{\theta}^{-1 / 6}
$$

Denton [2] noted reasonable agreement between Eq. (4) and data for $\operatorname{Re}_{\theta}$ between 500 and 1000 for accelerating, constant pressure, and diffusing boundary layers.

For the transition region no correlation exists to describe the distribution of the dissipation coefficient with varying $\operatorname{Re}_{\theta}$. Emmons [13] presumed that since the flow in the transition region is part of the time laminar $(\gamma=0)$ and part of the time turbulent $(\gamma$ $=1$ ), the average flow at any streamwise position is given by

$$
f=(1-\gamma) f_{\mathrm{LAM}}+\gamma f_{\mathrm{TURB}}
$$

Here $f$ is a boundary layer flow related quantity with $f_{\text {LAM }}$ and $f_{\text {TURB }}$ as its local laminar and fully turbulent values. Such a linear combination method, although relatively simple, has proven to be an effective and useful transition model. Dhawan and Narasimha [14] showed that this equation gives reasonable approximations for the boundary layer thicknesses and shape factor. Dhawan and Narasimha [14] also showed that the skin friction coefficient $C_{f}$ may be substituted into Eq. (5) to give good agreement with experimental data. Dey [21] has shown that the momentum imbalance due to the method is small enough to be negligible. The dissipation coefficient $C_{D}$ is inserted into Eq. (5) in a similar manner to yield

$$
C_{D}=(1-\gamma) C_{D_{\mathrm{LAM}}}+\gamma C_{D_{\text {TURB }}}
$$

This equation will be used to determine the intermittency weighted entropy generation rate in the transition region from the conditionally sampled data.

\section{Transition Region Experimental Data}

All the calculations presented in this paper are based on data presented by Volino et al. [17] in which detailed information regarding the experimental apparatus and method can be found. A brief summary of the experimental data is given here. The data are hot wire measurements from ten measurement stations that extend the length of the transition region of an accelerating boundary layer subject to a velocity gradient of $13.9 \mathrm{~s}^{-1}$. The streamwise free-stream turbulence intensity is $8.8 \%$ at the test section inlet and drops to $2 \%$ at station 10. Data were taken for $26 \mathrm{~s}$ at a $20 \mathrm{kHz}$ sampling rate for each measurement point using a boundary layer type hot wire probe, a boundary layer cross-wire probe, and a constant temperature hot wire anemometer control system. Distance from the wall is known to within $25 \mu \mathrm{m}$. The data were low-pass filtered at $10 \mathrm{kHz}$. Uncertainties in mean and rms fluctuating velocities are 3-5\%. Uncertainty in the Reynolds shear stresses $-\overline{u^{\prime} v^{\prime}}$ is $10 \%$. Uncertainty in skin friction coefficient $C_{f}$ is $8 \%$. Uncertainties in the momentum and displacement thicknesses are $10 \%$. Uncertainty in the shape factor $H_{12}$ is $7 \%$. Intermittency was calculated from both $u^{\prime}$ and $u^{\prime} v^{\prime}$ with good agree- 
Table 1 Boundary layer data [17]

\begin{tabular}{lccccccc}
\hline \hline & & & $\bar{u}_{\infty}^{\prime}$ & $\overline{v_{x}^{\prime}}$ & & & \\
Station & $\begin{array}{c}x \\
(\mathrm{~m})\end{array}$ & $\begin{array}{c}U_{\infty} \\
(\mathrm{m} / \mathrm{s})\end{array}$ & $\begin{array}{c}U_{\infty} \\
(\%)\end{array}$ & $\begin{array}{c}U_{\infty} \\
(\%)\end{array}$ & $\begin{array}{c}K \\
(\%)\end{array}$ & $\begin{array}{c}\gamma_{p k} \\
(\%)\end{array}$ & $\begin{array}{c}\delta_{99.5} \\
(\mathrm{~mm})\end{array}$ \\
\hline 1 & 0.1182 & 6.22 & 6.4 & 6.4 & 5.32 & 4.1 & 4.04 \\
2 & 0.1895 & 7.12 & 4.9 & 5.5 & 4.04 & 3.0 & 3.99 \\
3 & 0.2677 & 7.99 & 3.9 & 4.8 & 3.25 & 4.7 & 4.52 \\
4 & 0.3449 & 9.13 & 3.2 & 4.2 & 2.53 & 8.9 & 5.16 \\
5 & 0.4231 & 10.35 & 2.7 & 3.8 & 1.97 & 17.0 & 4.72 \\
6 & 0.5033 & 11.38 & 2.4 & 3.4 & 1.63 & 34.4 & 5.28 \\
7 & 0.5805 & 12.49 & 2.1 & 3.2 & 1.33 & 56.0 & 5.67 \\
8 & 0.6587 & 13.63 & 1.9 & 2.9 & 1.13 & 71.4 & 5.80 \\
9 & 0.7353 & 14.62 & 1.7 & 2.7 & 0.97 & 86.2 & 6.58 \\
10 & 0.8165 & 15.89 & 1.5 & 2.5 & 0.83 & 93.2 & 7.92 \\
\hline \hline
\end{tabular}

ment found between the two. The correlation coefficients between the $u^{\prime}$ and $-\overline{u^{\prime} v^{\prime}}$ data were above 0.9 for $95 \%$ of the measurement locations, with 0.8 as the lowest value. Uncertainty in the intermittency is $10 \%$. Volino et al. [17] refer to the laminar conditioned data as "nonturbulent" due to elevated fluctuations in the laminar region of transition and to the unconditioned data as "composite." A summary of the measurements at each station is given in Tables 1 and 2. Uncertainty in the laminar-conditioned dissipation coefficient data is calculated at $6 \%$. The uncertainties in turbulentconditioned and nonconditionally sampled dissipation coefficient data where the Reynolds shear stresses are included are calculated at $10 \%$.

\section{Entropy Calculations}

Figure 1 shows the mean velocity profiles for stations $1-9$ in wall coordinates. For the turbulent conditioned data there is good agreement with the Von Kármán empirical correlation. The Reynolds shear stresses for the turbulent conditioned data for measurement stations 1-9 are also shown in Fig. 1, nondimensionalized with the turbulent friction velocity $u_{\tau}^{2}$. The $-\overline{u^{\prime} v^{\prime}}$ fluctuations for the laminar conditioned data are significantly smaller than the turbulent conditioned data and have been neglected in the laminar dissipation coefficient calculations. The Reynolds shear stresses for the turbulent conditioned data and the nonconditionally sampled data were fitted with a sixth order polynomial set to zero at the wall. This was found to sufficiently fit the data. This polynomial equation was used to represent the Reynolds shear stresses in Eq. (1) for each of the mean velocity profiles. The peaks in the $-\overline{u^{\prime} v^{\prime}}$ profiles are around $Y^{+} \approx 30$, in broad agreement with classical data available in the literature, Roach and Brierley [22] with peak $-\overline{u^{\prime} v^{\prime}}$ found in $Y^{+} \approx 30-40$ for fully developed turbulent boundary layers. Some scatter is observed in the turbulent Reynolds shear stresses at low Reynolds numbers. This is due to the low intermittency level in this region.

Detailed near wall measurements are needed in order to calculate the dissipation coefficient accurately. The mean boundary layer velocity profiles were fitted with a linear approximation in the near-wall region $\left(Y^{+}=U^{+}\right)$with an overlapping sixth order polynomial to the remainder of velocity profile. This method is considered to be more accurate than applying a single high order polynomial fit as it exploits the law of the wall in the viscous sublayer where $Y^{+}=U^{+}$. It was found that the overlap between the linear and nonlinear portions of the velocity profiles was at approximately $Y^{+} \approx 5( \pm 2)$, in agreement with the "universal law" of the wall. This result gives confidence in the validity of the measurements and the methodology proposed to determine the entropy generation rate. The velocity profile curve fits are also set to zero at the wall. The entropy generation rates calculated for station 5 are shown in Fig. 2. A large difference between the laminar conditioned data and the total turbulent conditioned data is visible. Using Eq. (1) the total turbulent nondimensional entropy generation rate can be calculated by summing the viscous and Reynolds shear stress contributions. The flat regions of both the laminar and turbulent conditioned viscous data are a result of the linear curve fits where $\partial u / \partial y$ is constant. Since the linear curve fit of the velocity profile must pass through the origin in accordance with the zero slip condition, the linear curve fit may be forecasted back to the wall when integrating the curves to determine the dissipation coefficient.

The conditionally-sampled and nonconditionally sampled $\dot{S}_{\text {gen }}^{\prime \prime \prime}$ plots for all the measurement stations given in Tables 1 and 2 are shown in the form of nondimensional contour plots in Fig. 3. $\dot{S}_{\text {gen }}^{\prime \prime \prime}$ is nondimensionalized by $\left(\dot{S}_{\text {gen }}^{\prime \prime \prime}\right) *=\dot{S}_{\text {gen }}^{\prime \prime \prime} \theta T / \rho U_{x}^{3}$, where the length scale $\theta$ is the nonconditionally-sampled momentum thickness. The plots are smoothed with a spline interpolation between data points.

Figure 3 shows (a) laminar data which represents the flow between the turbulent spots, (b) turbulent data which represent the flow in the turbulent spots, and (c) intermittently weighted data, which are intermittency weighted representations of the flow field at each measurement station calculated by inserting the data from

Table 2 Conditionally sampled boundary layer data [17]

\begin{tabular}{|c|c|c|c|c|c|c|}
\hline \multirow[b]{2}{*}{ Station } & $\operatorname{Re}_{\theta}$ & $H$ & $\operatorname{Re}_{\theta}$ & $H$ & $\operatorname{Re}_{\theta}$ & $H$ \\
\hline & \multicolumn{2}{|c|}{ Composite } & \multicolumn{2}{|c|}{ Nonturbulent } & \multicolumn{2}{|c|}{ Turbulent } \\
\hline 1 & 136 & 1.96 & 136 & 1.97 & 90 & 1.97 \\
\hline 2 & 149 & 1.98 & 149 & 1.99 & 135 & 1.72 \\
\hline 3 & 169 & 1.94 & 168 & 1.94 & 186 & 1.59 \\
\hline 4 & 196 & 1.87 & 193 & 1.89 & 244 & 1.51 \\
\hline 5 & 221 & 1.82 & 212 & 1.86 & 293 & 1.48 \\
\hline 6 & 263 & 1.76 & 239 & 1.86 & 366 & 1.46 \\
\hline 7 & 324 & 1.66 & 264 & 1.86 & 432 & 1.46 \\
\hline 8 & 372 & 1.58 & 278 & 1.82 & 474 & 1.43 \\
\hline 9 & 457 & 1.49 & 297 & 1.81 & 580 & 1.39 \\
\hline 10 & 580 & 1.43 & 364 & 1.69 & 745 & 1.35 \\
\hline
\end{tabular}



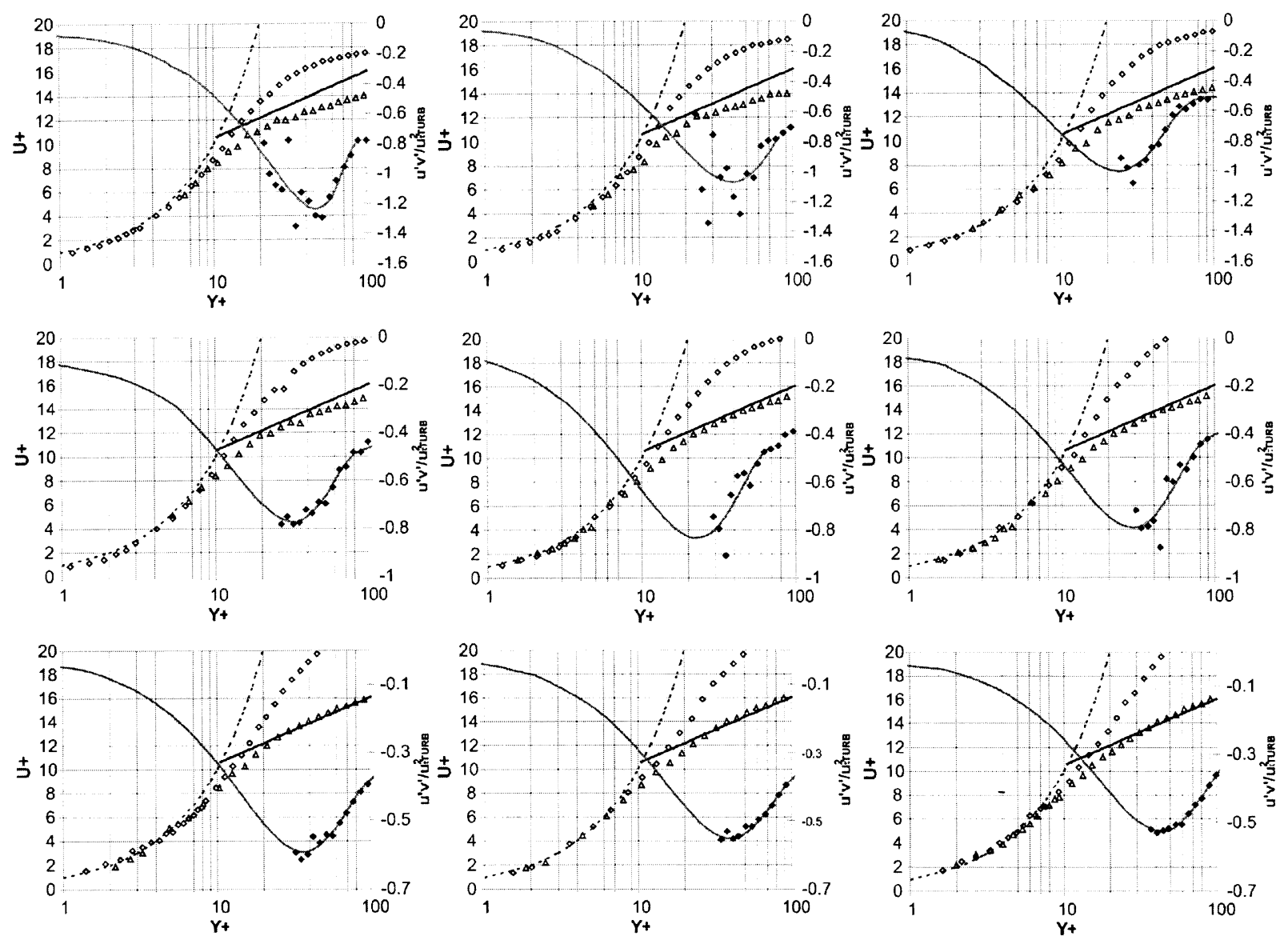

Fig. 1 Conditionally-sampled velocity profiles and Reynolds shear stresses for stations 1-9: $\bigcirc$, laminar-conditioned data; $\triangle$ turbulent-conditioned data; $\downarrow$, turbulent-conditioned Reynolds shear stresses; ---, law of the wall, $\gamma^{+}=U^{+} ;-$, Von Kármán empirical correlation $U^{+}=2.4 \ln Y^{+}+5$; - , sixth order polynomial fit of turbulent-conditioned Reynolds shear stress data

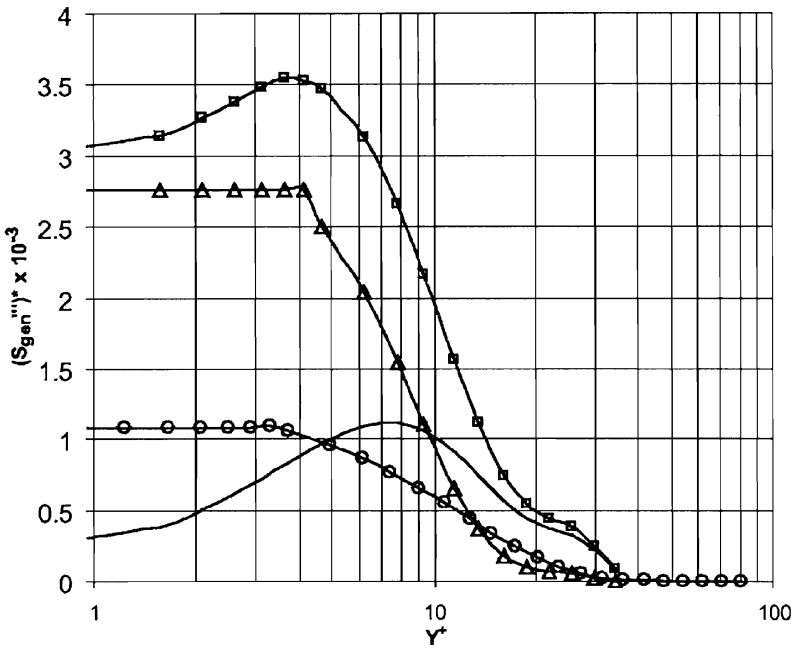

Fig. 2 Entropy generation rate profiles for station 5: 0 , laminar-conditioned data; $\triangle$, viscous turbulent conditioned data; - Reynolds shear stress turbulent-conditioned data; $\square$, total turbulent-conditioned data
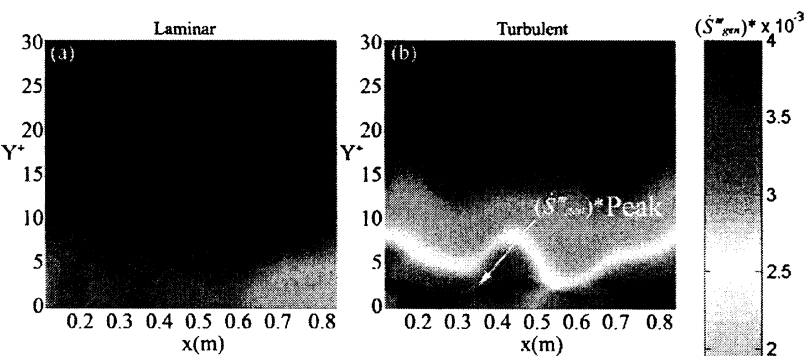

Conditionally-Sampled
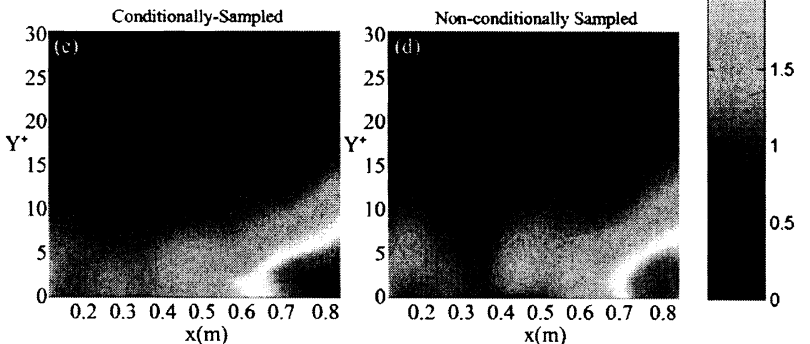

Fig. 3 Contours of nondimensional volumetric entropy generation rate for (a) laminar conditionally-sampled, $(b)$ turbulent conditionally-sampled, (c) intermittency weighted data, and (d) nonconditionally sampled 


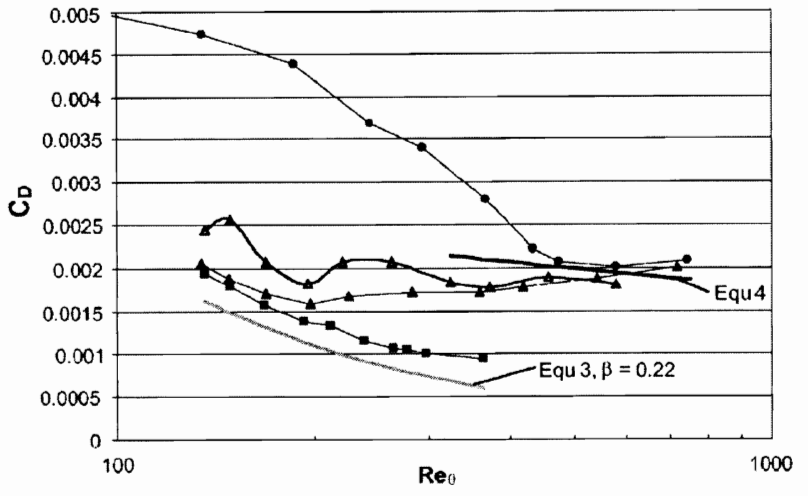

Fig. 4 Dissipation coefficient vs $\operatorname{Re}_{\theta}$ : $\mathbf{\square}$, laminar-conditioned data; $\bullet$, turbulent-conditioned data (note: station 1 data point for turbulent-conditioned data are located at $\mathbf{R e}_{\theta}=90$ ); $\boldsymbol{\Lambda}$, intermittency weighted data; $\triangle$, unconditionally sampled data

(a) and (b) into Eq. (6). A nonconditionally sampled, timeaveraged picture of the flow field (d) is also shown.

The dissipation coefficient, Eq. (2), results obtained for each measurement station given in Tables 1 and 2 are shown in Fig. 4 . This figure is obtained by integrating the contours of Fig. 3 across the boundary layer thickness at each measurement station. The data are presented in a similar manner to Fig. 3. with the laminarconditioned data and turbulent-conditioned data representing the flow conditions due to intermittent turbulent spot passage and the intermittency weighted and nonconditionally sampled data showing the difference between the intermittency weighted and time averaged interpretations of the flow field. The data are plotted against $\operatorname{Re}_{\theta}$ for the respective data set. $\operatorname{Re}_{\theta}$ for the intermittently averaged data were calculated by applying Eq. (5) to the $\operatorname{Re}_{\theta}$ values for laminar and turbulent conditionally sampled data. The existing laminar and turbulent correlations, Eqs. (3) and (4), are included as references in Fig. 4.

\section{Discussion}

High levels of entropy generation rates per unit volume in the near wall region are a common feature found in the literature $[1,2,20]$ and are present in the current data as seen in Fig. 3. This trend is found in both the laminar and turbulent profiles throughout the transition region. From the entropy generation profiles in the turbulent region of Fig. 2 and Fig. 3(b), we find a consistent peak entropy generation rate per unit volume away from the wall in the region $2 \leqslant Y^{+} \leqslant 3$; this peak is a result of the linear velocity profile up to the $Y^{+} \approx 5$ region combined with increasing Reynolds stresses in this region. This visibility of the peak in Fig. $3(b)$ is enhanced with the addition of a 3D light source. The peak associated with station 5 occurs at $Y^{+}=4$ as a result of a shallower entropy generation rate profile. This shallower profile is found to be a result of the higher Reynolds shear stress contribution to the entropy generation rate for station 5 . Unfortunately conditionally sampled data are rare in the literature preventing a comparison of this behavior with other such data.

For the laminar-conditionally sampled data of Fig. 4, we find a trend of decreasing dissipation coefficient with increasing Reynolds number based upon the momentum thickness, which is in qualitative agreement with the long standing correlation of Truckenbrodt [3], Eq. (3), also shown in Fig. 4. In terms of magnitude, with a value of $\beta=0.22$, which is the maximum using the limits imposed on the pressure gradient parameter by Schlichting [5], the curve falls consistently $25 \%$ below the measured data. The reason for this may be that the acceleration parameter in the current data is greater than the maximum allowed by the Pohlhausen family of velocity profiles from which the $\beta$ value is obtained. It is likely that it is also partly due to the laminar region directly behind a turbulent spot where the dissipation coefficient levels are high before relaxing to the laminar level; readjusting after the turbulent spot has passed the measurement location. Either way calculating $\beta$ using the Pohlhausen pressure gradient shape factor $\Lambda$ for the current data, as described by Denton [2], gives $\beta=0.27$ giving excellent agreement with the current data, with an average difference from measurements of less than $5 \%$.

In the conditionally sampled turbulent data very high levels of dissipation coefficient are deduced in the region of low intermittency. These high dissipation levels may be due to the high velocity gradients found in a relatively thin turbulent boundary layer. At low momentum thickness Reynolds numbers the magnitude of the values are approximately twice those correlated by Schlichting [5], Eq. (4). However as the intermittency increases we find good agreement with the correlation of Schlichting [5] at $\operatorname{Re}_{\theta}>500$ in agreement with the observations of Denton [2].

There is a large amount of scatter observed in the turbulent conditioned Reynolds shear stresses at stations 1 and 2 where the intermittency is between $3 \%$ and $4 \%$. As a result very little turbulent data are recorded during the $26 \mathrm{~s}$ sampling time. Perhaps better results may be obtained if the sampling time is selected based on the intermittency factor to ensure adequate sampling of such short scale temporal events. Such measurements are further hampered by the relatively bulky cross wire probes used to measure the Reynolds shear stresses in a thin accelerating unsteady boundary layer.

The intermittency weighted data are a combination of the conditionally sampled laminar and turbulent flow, Eq. (6), and are the first such representation of the dissipation coefficient in the transition region. It is found that the dissipation coefficient follows a similar trend to that of the widely employed skin friction coefficient in the transition region and thus presents a number of established techniques to allow its prediction. The difficulty lies in predicting the laminar and turbulent dissipation coefficient distributions initially. This task is made more difficult by the limited amount of these types of measurements available in the literature.

For the nonconditionally sampled data of Fig. 4 there is up to a $20 \%$ higher dissipation coefficient calculated at low Reynolds numbers when compared to the intermittency weighted conditionally sampled data. Two distinct peaks are seen in the distribution of the dissipation coefficient. Similar peaks were also found in the time averaged data of Stieger [19] and O'Donnell [23]. As shown above, these peaks are in fact not representative of the dissipation coefficient in a transitional boundary layer and are misleading in determining the sources of thermodynamic loss. The peaks are caused by the time averaging technique employed in the data processing, where large Reynolds stresses are inferred from measurements. These large fluctuations are not the result of local Reynolds stresses, but due to the intermittent nature of the boundary layer. Hence these fluctuations do not contribute to the entropy generation rate because they are short term temporal events and cannot simply be time averaged. Furthermore as the conditioned and nonconditioned data imply the time averaging approach is particularly inappropriate in regions of low intermittency. This is unfortunate as most of the existing data are of the time averaged, and not conditionally sampled type.

In summary, this paper presents the first look at the entropy generation rate in a conditionally sampled transitional boundary layer. This is important, because it is the first step towards developing a predictive technique for the entropy generation rate in the transition region to accompany the long-standing laminar and turbulent correlations. Such correlations are necessary for the advancement of boundary layer codes.

\section{Conclusions}

In this paper entropy generation measurements in a transitional boundary layer weighted on intermittency have been calculated successfully for the first time. There is a significant difference 
between the time averaged data with the intermittency weighted data in both magnitude and distribution with up to a $20 \%$ deviation between the two. A more gradual transition from the laminar to the turbulent state is seen in the conditionally sampled data rather than the somewhat erratic time averaged data, which exhibited a double peak caused by the passing of turbulent spots over the sensor. Agreement with the trend of the laminar correlation of Truckenbrodt [3] is reasonable for all Reynolds numbers considered but the experimental data is consistently $25 \%$ higher than the prediction. Calculating the Pohlhausen pressure gradient shape factor for the current data gives a value of $\beta=0.27$, and gives excellent agreement with the experimental data, although this $\beta$ value is outside the recommended range of the Truckenbrodt correlation. The turbulent correlation also agrees well with the data in the turbulent region. In summary, using this technique with conditionally sampled data, a more accurate representation of the thermodynamic losses is made possible and is the first step in code development for predicting the thermodynamic loss in the transition region.

\section{Acknowledgment}

This publication has emanated from research conducted with the financial support of the Science Foundation of Ireland. The INL contribution was partly supported via DOE Idaho Operations Office Contract No. DE-AC07-05ID14517.

\section{Nomenclature}

$C_{D}=$ dissipation coefficient

$C_{f}=$ skin friction coefficient

$H_{12}=$ boundary layer shape factor $\left(\delta^{*} / 0\right)$

$K=$ acceleration parameter

$\operatorname{Re}_{\theta}=$ momentum thickness Reynolds number

$\operatorname{Re}_{x}=$ streamwise coordinate Reynolds number

$\dot{S}^{\prime \prime \prime}=$ entropy generation rate per unit volume, $\mathrm{W} \mathrm{m} \mathrm{m}^{-3} \mathrm{~K}$

$T=$ absolute temperature, $\mathrm{K}$

$u=x$ direction velocity, $\mathrm{m} \mathrm{s}^{-1}$

$U_{e}=$ boundary layer edge velocity $\left(0.99 U_{\infty}\right), \mathrm{m} \mathrm{s}^{-1}$

$U_{-\infty}=$ free-stream velocity, $\mathrm{m} \mathrm{s}^{-1}$

$u^{\prime}=$ instantaneous streamwise fluctuating velocity, $\mathrm{m} \mathrm{s}^{-1}$

$U^{+}=$local mean streamwise velocity in wall coordinates

$u_{\tau}=$ friction velocity, $\mathrm{m} \mathrm{s}^{-1}$

$v^{\prime}=$ instantaneous cross-stream fluctuating velocity, $\mathrm{m} \mathrm{s}^{-1}$

$x=$ streamwise coordinate, distance from the leading edge, $m$

$y=$ cross-stream coordinate, distance from the wall, $\mathrm{m}$

$Y^{+}=$distance from the wall in wall coordinates

\section{Greek Symbols}
$\delta=$ boundary layer thickness, $\mathrm{m}$
$\delta^{*}=$ displacement thickness, $\mathrm{m}$
$\mu=$ dynamic viscosity, $\mathrm{N} \mathrm{s} \mathrm{m}^{-2}$
$\Lambda=$ Pohlhausen pressure gradient shape factor
$\gamma=$ intermittency

$$
\begin{aligned}
& \theta=\text { momentum thickness, } \mathrm{m} \\
& \rho=\text { density, } \mathrm{kg} \mathrm{m}^{-3} \\
& \nu=\text { kinematic viscosity, } \mathrm{m}^{2} \mathrm{~s}^{-1}
\end{aligned}
$$

\section{Subscripts}

LAM = laminar condition

TURB $=$ turbulent condition

\section{References}

[1] Bejan. A., 1982, Entropy Generation Through Heat and Fluid Flow, Wiley, NY.

[2] Denton, J. D., 1993, "Loss Mechanisms in Turbomachines," ASME J. Turbomach.. 115, pp. 621-656.

[3] Truckenbrodt, E., 1952, "A Method of Quadrature for the Calculation of the Laminar and Turbulent Boundary Layer in Case of Plane and Rotational Symmetric Flow," NACA TM 1379, 1955 (Translated as Ing.-Arch., 20(4), pp. 211-228).

[4] Pohlhausen, K., 1921, "Zur Näherungsweisen Integration der Differentialgieichung der Laminaren Reibungsschicht," Z. Angew. Math. Mech., 1, 252-268.

[5] Schlichting, H., 1979, Boundary Layer Theory, 7th ed., Mc-Graw Hill, NY.

[6] Walsh, E. J., and Davies, M. R. D., 2005, "Measurements in the Transition Region of a Turbine Blade Profile Under Compressible Conditions," ASME J. Fluids Eng., 127, pp. 400-403.

[7] Mayle, R. E., 1991, "The Role of Laminar-Turbulent Transition in Gas Turbine Engines," ASME J. Turbomach., 113. pp. 509-537.

[8] Walsh, E. J., and Davies, M. R. D., 2003 "Measurement and Prediction of Transition on the Suction Surface of Turbine Blade Profiles," Proceedings of the 5th European Conference on Turbomachinery. Fluid Dynamics and Thermodynamics. Prague, Czech Republic, Paper No. TT01-201.

[9] Gostelow, J. P., Blunden, A. R., and Walker, G. J., 2004 "Effects of Free Stream Turbulence and Adverse Pressure Gradients on Boundary Layer Transition," ASME J. Turbomach., 116, pp. 392-404.

[10] Boyle, R. J., and Simon, F. F., 1999, "Mach Number Effects on Turbine Blade Transition Length Prediction," J. Turbomach., 121, pp. 694-702.

[11] Suzen. Xiong. Y.B., G., and Huang, P. G., 2002, "Predictions of Transitional Flows in Low-Pressure Turbines using Intermittency Transport Equation," AIAA J., 40, pp. 254-266.

[12] Abu-Ghannam, B. J., and Shaw, R., 1980, "Natural Transition of Boundary Layers-The Effect of Turbulence, Pressure Gradient and Flow History," J. Mech. Eng. Sci., 22, pp. 213-228.

[13] Emmons, H. W., 1951, "The Laminar-Turbulent Transition in a Boundary Layer," J. Aeronaut. Sci., 18, pp. 490-498.

[14] Dhawan, S., and Narasimha, R., 1958, "Some Properties of Boundary Layer Flow During the Transition From Laminar to Turbulent Motion," J. Fluid Mech., 3, pp. 418-436.

[15] Kim, J., and Simon, T. W., 1991, "Free-Stream Turbulence and Concave Curvature Effects on Heated, Transitional Boundary Layers" Final Report, Minnesota University, Minneapolis, Department of Mechanical Engineering, Vol. I, NASA CR 187150 and Vol. II, NASA CR 187151.

[16] Wang, T., and Zhou, D., 1998, "Conditionally Sampled Flow and Thermal Behaviour of a Transitional Boundary Layer at Elevated Free-Stream Turbulence," Int. J. Heat Fluid Flow, 19, pp. 348-357.

[17] Volino, R. J., Schultz, M. P., and Pratt, C. M.. 2003, "Conditional Sampling in a Transitional Boundary Layer under High Free-Stream Turbulence Conditions," ASME J. Fluids Eng., 125, pp. 28-37.

[18] Schobeiri, M. T., Read. K., and Lewalle, J., 2003, "Effect of Unsteady Wake Passing Frequency on Boundary Layer Transition, Experimental Investigation, and Wavelet Analysis," ASME J. Fluids Eng., 125, pp. 251--266.

[19] Stieger, R. D., 2002, "The Effects of Wakes on Separating Boundary Layers in Low Pressure Turbines," Ph.D. dissertation, Engineering Department, Cambridge University, Cambridge, UK.

[20] Kim, J.. Simon, T. W., and Kestoras, M., 1994, "Fluid Mechanics and Heat Transfer Measurements in Transitional Boundary Layers Conditionally Sampled on Intermittency," ASME J. Turbomach., 116, pp. 405-416.

[21] Dey, J., 2000, "On the Momentum Balance in Linear-Combination Models for the Transition Zone," ASME J. Turbomach., 122, pp. 587-588.

[22] Roach, P. E., and Brierley, D. H., 1990, "The Influence of a Turbulent Free Stream on Zero Pressure Gradient Transitional Boundary Layer Development. Part 1: Test Cases T3A and T3B," Numerical Simulation of Unsteady Flow's and Transition to Turbulence, Cambridge University Press, pp. 319-347.

[23] O'Donnell, F. K., 2000, "The Measurement of Aerodynamic Entropy Generation in a Turbine Blade Boundary Layer," Ph.D. thesis, Mechanical and Aeronautical Department, University of Limerick, Ireland. 